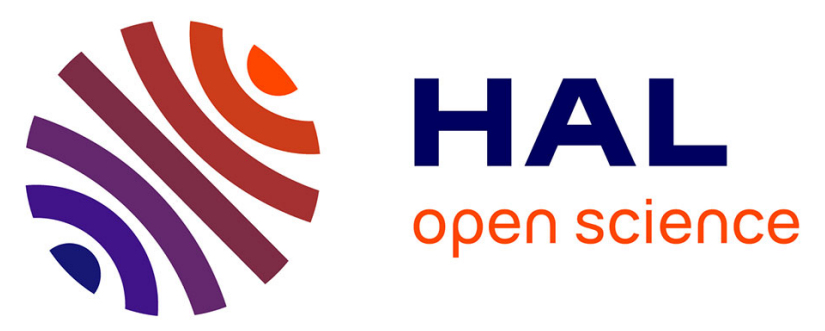

\title{
La place des monuments publics du Haut-Empire dans les villes de la Gaule méridionale durant l'Antiquité tardive (IVe-VIe s.)
}

Marc Heijmans

\section{- To cite this version:}

Marc Heijmans. La place des monuments publics du Haut-Empire dans les villes de la Gaule méridionale durant l'Antiquité tardive (IVe-VIe s.). Gallia - Archéologie de la France antique, 2006, Antiquité tardive, haut Moyen Âge et premiers temps chrétiens en Gaule méridionale (première partie) : réseau des cités, monde urbain et monde des morts, 63, pp.25-41. 10.3406/galia.2006.3281 . hal01914286

\section{HAL Id: hal-01914286 \\ https://hal.science/hal-01914286}

Submitted on 8 Jan 2020

HAL is a multi-disciplinary open access archive for the deposit and dissemination of scientific research documents, whether they are published or not. The documents may come from teaching and research institutions in France or abroad, or from public or private research centers.
L'archive ouverte pluridisciplinaire HAL, est destinée au dépôt et à la diffusion de documents scientifiques de niveau recherche, publiés ou non, émanant des établissements d'enseignement et de recherche français ou étrangers, des laboratoires publics ou privés.

\section{(ㅇ)(1) $\$$}

Distributed under a Creative Commons Attribution - NonCommercial - NoDerivatives $\mid 4.0$ 


\title{
LA PLACE DES MONUMENTS PUBLICS DU HAUT-EMPIRE DANS LES VILLES DE LA GAULE MÉRIDIONALE DURANT L'ANTIQUITÉ TARDIVE (IV ${ }^{\mathrm{e}}-\mathrm{VI}^{\mathrm{e}} \mathrm{S}$.)
}

\author{
Marc HEIJMANS
}

\begin{abstract}
Mots-clés. Législation, temple, forum, monument de spectacle, thermes, église, réoccupation.
Résumé. Le plus souvent, le rôle des monuments publics du Haut-Empire dans les villes de l’Antiquité tardive se concentre sur leur réutilisation en tant qu'église. Après un rappel du cadre juridique, qui montre que jusqu'à la fin du IVe s. on a essayé de maintenir en état les édifices publics, y compris les temples, l'étude de ces monuments, catégorie par catégorie, pour le sud de la Gaule, confirme cette position, en tout cas pour ceux situés au centre des villes tardives; ce n'est que depuis la fin du IVe s. que ces monuments sont également spoliés ou transformés dans un monde devenu chrétien, où le maintien des parures de la ville classique n'avait plus la priorité. La transformation d'un temple en église est cependant exceptionnelle.
\end{abstract}

Key-words. Legislation, temple, forum, entertainment building, thermae, church, reoccupation.

Abstract. Very often, the purpose of public monuments from the Principate, in the towns of late Antiquity concerns their re-utilization as a church. Following a reminder of the legal context showing that public buildings were kept in state, even temples, the study of these buildings being categorized in southern Gaul confirms this situation, at least for those located in the centre of the late towns. By the end of the $4^{\text {th }}$ c. only, these monuments became spolia or were transformed within a new Christian world where the preservation of the urban ornaments was not anymore a priority. However, the case of a temple converted into a church does not often happen.

Translation: Isabelle FAUDUET

Schlagwörter. Gesetzgebung, Tempel, forum, Theaterbau, Thermen, Kirche, Umnutzung.

Zusammenfassung. In den spätantiken Städten konzentriert sich die Rolle der öffentlichen Großbauten aus der mittleren Kaiserzeit in den meisten Fällen auf ihre Wiederverwendung als Kirchen. Der Beitrag erinnertt zunächst an den bekannten Gesetzesrahmen und zeigt, daß man bis ans Ende des vierten Jahrhunderts die öffentlichen Bauten, und zwar sogar die Tempel, instandzuhalten versuchte. Eine systematische Untersuchung der betreffenden Großbauten im Süden Galliens bestätigt diese Einschätzung, zumindest was die Anlagen in den Zentren der spätantiken Städte angeht. Erst ab dem Ende des vierten Jahrhunderts greift man auch auf solche Großbauten zur Entnahme von Bauteilen zu oder wandelt sie um. Dies geschieht vor dem Hintergrund einer christianisierten Lebenswelt, in der die Pflege der Pracht der klassischen Stadt keine Bedeutung mehr hat. Die Umwandlung eines Tempels in eine Kirche bleibt aber die Ausnahme.

Übersetzung: Stefan WIRTH

La transformation de la ville entre l'Antiquité tardive et le haut Moyen Âge a fait l'objet d'une attention soutenue ces dernières années. Nombreux sont les colloques, congrès et monographies consacrés à ce sujet, et l'archéologie urbaine est désormais plus attentive aux niveaux, parfois ténus et peu spectaculaires, que livrent les fouilles pour l'Antiquité tardive.
Cette attention pour la ville de l'Antiquité tardive n'est pas nouvelle en soi, mais a d'abord été consacrée au christianisme et son apport à la topographie, dont témoigne la série, presque achevée, de la Topographie chrétienne des cités de la Gaule (TCCG, I-XIII, 1986-2004). Dans le même esprit, qui voyait surtout dans l'Antiquité tardive le rôle de la nouvelle religion, la question de savoir ce que sont 
devenus les édifices hérités de l'Antiquité classique s'est généralement limitée à leur transformation en lieux de culte chrétiens, et notamment les temples païens (Hanson, 1978; Vaes, 1989).

La survie des autres édifices de l'Antiquité classique a moins retenu l'attention des chercheurs et les études régionales sont peu nombreuses, comme l'a souligné encore récemment W. Liebeschuetz (2001, p. 30). Certes, en 1974, P.-A. Février a consacré une importante étude à cette question (Février, 1974), notamment pour la partie occidentale du bassin méditerranéen, tandis que C. Lepelley a analysé quelques années plus tard la survie des cités et de leurs institutions en Afrique du Nord, toutefois essentiellement par l'analyse les sources littéraires et épigraphiques (Lepelley, 1979-1981). Plus limitée géographiquement a été l'étude de Bryan Ward-Perkins, consacrée à l'Italie du Nord (Ward-Perkins, 1984), ou des études récentes sur l'Espagne (Jiménez Sanchez, Sales Carbonell, 2004), mais pour le midi de la Gaule il manque un survol récent des données archéologiques.

Le but de notre étude est de faire, de façon plus modeste, un bilan de ce legs des villes antiques aux civitates du midi de la Gaule durant l'Antiquité tardive. Elle est largement basée sur l'analyse de publications récentes, heureusement nombreuses, qu'il s'agisse de mises au point régionales, comme les colloques sur l'Aquitaine (Maurin, Pailler dir., 1996), ou de monographies sur une ville, comme Aix-enProvence (Guyon et al., 1998), Arles (Heijmans, 2004a), Fréjus (Rivet et al., 2000), Marseille (Bouiron, Tréziny dir., 2001), Nîmes (Monteil, 1999) ou Toulouse (Pailler dir., 2001). Enfin, les fascicules de la Carte archéologique de la Gaule, là où ils existent, ont parfois permis de compléter les renseignements. Contrairement à l'Italie, les sources littéraires sont plus rares et se réduisent pour l'essentiel à des observations tirées de l'épigraphie ou de l'hagiographie, encore mal exploitées dans ce domaine.

\section{LE CADRE JURIDIQUE}

Un certain nombre de lois concernant les édifices publics ont été conservées dans les grandes collections juridiques de la fin de l'Antiquité, notamment dans les livres XV, XVI et XVI, $10 \mathrm{du}$ Code théodosien (C. th.) ${ }^{57}$. Elles témoignent de la volonté de l'État de préserver, autant que possible, les anciens monuments, mais également de la pression des particuliers, qui souhaitaient s'emparer de ces construc-

57. Pour un ensemble des constitutions sur les édifices publics, voir Janvier, 1969; pour les temples païens, voir Magnou-Nortier, 2002. tions. Ainsi, une loi de 357 interdit le dépouillement des décors architecturaux des édifices publics, sans doute des colonnes ou des placages de marbre, car « il n'est pas juste qu'une cité perde la parure reçue des aïeux » (C. th., XV, $1,1)$. La législation est cependant encore tolérante envers les particuliers qui ont construit des maisons sur des domaines publics, car sous Julien, ils pouvaient garder ces constructions de plein droit (C. th., XV, 1, 8-9). Plusieurs lois de la seconde moitié du $\mathrm{IV}^{\mathrm{e}} \mathrm{s}$., promulguées en Occident comme en Orient, montrent la priorité donnée à la restauration des édifices ruinés par la vétusté (C. th., XV, 1, 11 [364] ; XV, 1, 15 [365] ; XV, 1, 16 [365] ; XV, 1, 17 [365] ; XV, 1, 19 [376] ; $\mathrm{XV}, 1,20$ [380] ; XV, 1, 21 [380] ; XV, 1, 27 [390] ; XV, 1, 29 [393] ; XV, 1, 32 [395] ; XV, 1, 33 [395]). En même temps cependant, il est interdit de rechercher des matériaux de construction dans des villages désertés afin d'embellir la métropole ou les cités les plus importantes (C. th., XV, 1, 14 [365]) ou des monuments publics (C. th., XV, 1, 19 [376]). Il est intéressant d'observer que l'argument avancé pour l'entretien des monuments antiques est souvent leur aspect esthétique (Brenk, 1987).

À la fin du $\mathrm{IV}^{\mathrm{e}} \mathrm{s}$. cependant, le problème des constructions « sauvages " suscite à nouveau l'attention des pouvoirs publics, notamment en Orient. Ainsi, en 383, une loi adressée au comte d'Orient ordonne la démolition des constructions édifiées sur des lieux publics (C. just., VIII, $11,6)^{58}$. En 398, plusieurs lois ont été promulguées en Orient concernant cette question; deux d'entre elles ordonnent la destruction des constructions appuyées sur les entrepôts publics (C. th., XV, 1, 38) ou sur d'autres bâtiments publics ou privés, notamment pour des raisons de salubrité publique (C. th., XV, 1, 39 ; cf. XV, 1, 45-46 [406]). Une troisième loi stipule qu'un édifice public peut être aliéné au profit d'un particulier seulement s'il est en ruine ou de peu d'utilité pour les cités (C. th., XV, 1, 40). Les mêmes raisons sont évoquées en 398 pour interdire le dépouillement des décors de bronze ou de marbre (C. th., XV, 1, 37). La pression foncière des particuliers sur les édifices publics - et les temples - apparaît clairement à Constantinople, où toute une série de lois concerne des constructions privées qui se sont développées à l'intérieur des édifices publics, et en particulier du Palais impérial (C. th., XV, 1, 45-46 [406] ; XV, 1, 47 [409] ; XV, 1, 52 [424] ; C. just., XI, 77, 1 [426-429] ; VIII, 11, 20 [429] ; VIII, 21 [440]), mais elle était également présente en Occident, comme en témoignent des lois des années 400-405 qui visent à protéger

58. Voir C. th., XV, 1, 25, du 17 juillet 389, adressée au préfet de la ville de Constantinople. 
la propriété publique, tout en précisant que si le bien réclamé n'est pas utile à la cité ni ne contribue à sa parure, il peut être cédé à un particulier (C. th., X, 3, 5; XV, 1, 41 ; $\mathrm{XV}, 1,43)$. Visiblement, les lois n'ont pas été strictement appliquées, puisque au milieu $\mathrm{du} \mathrm{V}^{\mathrm{e}} \mathrm{s}$. les empereurs Léon et Majorien doivent constater, pour Rome, que la grande majorité des édifices antiques ont été détruits et que les pierres ont été réutilisées, souvent avec l'accord des pouvoirs publics, pour des constructions privées, au détriment de la splendeur des villes (splendor urbium). Ils interdisent donc la destruction des édifices - dont les temples - et prévoient des sanctions graves à l'encontre des magistrats qui osent permettre la profanation des monuments antiques, qui ne peuvent plus être cédés à des particuliers (Nov. Maj., IV [458]). Bien que ce constat ne concerne que Rome, il n'est guère douteux que, dans les villes provinciales, l'état de délabrement a dû être assez avancé et que l'intervention sévère des empereurs est manifestement venue trop tard.

Cette dernière loi, comme d'autres citées plus haut, ne fait aucune exception pour les temples paiens, ce qui peut paraître surprenant au premier abord. En réalité, la politique impériale à ce sujet est assez ambiguë. Durant la majeure partie du IV ${ }^{\mathrm{e}}$ s., les lois promulguées ont certes interdit les sacrifices aux dieux païens, mais les temples ont été préservés. Eusèbe de Césarée mentionne deux lois promulguées par Constantin contre le paganisme (Vie de Constantin, II, 45) et Jérôme signale dans sa Chronique qu'en 331, l'empereur a publié un édit ordonnant la destruction des temples, mais ces textes n'ont pas d'écho dans les recueils législatifs et leur application paraît douteuse. En 342 , on protège explicitement les temples situés hors des remparts, certes non pas pour des raisons religieuses, mais parce qu'ils sont à l'origine de jeux publics, qu'il ne faut manifestement pas annuler pour ne pas offenser le peuple (C. th., XVI, 10, 3). Quelques années plus tard, sans que la date soit certaine (Magnou-Nortier, 2002, p. 370-371), une loi ordonne la fermeture des temples, mais uniquement dans le but d'éviter que l'on y fasse des sacrifices (C.th., XVI, 10, 4). Sous Julien, la construction de nouveaux temples est autorisée (C. th., XV, 1,3) et encore en 382, le temple d'Édesse est maintenu ouvert par décret impérial (C. th., XVI, 10,8). Ce n'est qu'à la fin du IV ${ }^{\mathrm{e}} \mathrm{s}$., du fait de la politique anti-païenne de Théodose, que les premières lois visant les temples sont promulguées. Il faut en effet attendre 397 pour trouver une loi qui permet d'utiliser les matériaux provenant de la démolition des temples pour la réparation des voies, des aqueducs et des remparts (C. th., XV, 1, 36).
Dans les années 390, en effet, les lois deviennent plus strictes, en rappelant l'interdiction d'accéder aux temples et d'y célébrer des sacrifices (C. th., XVI, 10, 10 [391], XVI, 10, 11 [391], XVI, 10, 13 [395]) et si, en 399, une première loi demande la préservation des ornements des temples publics (C. th., XVI, 10, 15), une autre loi promulguée plus tard dans l'année ordonne, pour la première fois, la démolition des temples, mais uniquement ceux situés à la campagne, sans perturber la paix (C.th., XVI, 10, 16), puisque, au même moment, Honorius et Arcadius mettent en garde ceux qui souhaitent détruire les temples abandonnés (C. th., XVI, 10, 17). Au début du V ${ }^{\mathrm{e}}$ s., les temples sont confisqués par une loi de 407 et doivent être transformés pour un usage public (C. th., XVI, 10, 19), mais ce n'est qu'en 435, sous Valentinien III, que l'on voit pour la première fois la volonté de détruire les temples et de les affecter au culte chrétien (C. th., XVI, 10, 25). On a vu cependant qu'en 458, une autre loi interdit en revanche, la démolition des édifices publics encore debout à Rome, parmi lesquels les temples (Nov. Maj., IV).

En résumé, on peut constater que les législateurs romains ont essayé durant tout le $\mathrm{IV}^{\mathrm{e}} \mathrm{s}$. de maintenir en état les édifices publics des siècles précédents, certes plus pour le décor urbain et la gloire des ancêtres que pour leur utilité. Les temples païens ont été conservés, surtout en ville et si leur accès était interdit, afin d'éviter qu'on n'y pratique des sacrifices, jamais la politique impériale n'a visé la destruction systématique des temples avant une date avancée du $\mathrm{V}^{\mathrm{e}} \mathrm{s}$. Regardons à présent si les données archéologiques du midi de la Gaule concordent avec l'évolution théorique que nous dressent les lois.

\section{LES TEMPLES}

Comme nous l'avons signalé en introduction, dans la vision traditionnelle que l'on se forme du passage des villes antiques vers les villes médiévales, l'idée qui veut que les temples aient systématiquement donné naissance à une église chrétienne, ou, inversement, que sous chaque cathédrale se trouve un temple d'Auguste, de Jupiter ou de Diane, a la vie dure depuis les travaux de E. Mâle (1950). C'est encore la conclusion de Jan Vaes dans une étude présentée en 1986 au XI $\mathrm{X}^{\mathrm{e}}$ congrès international d'archéologie chrétienne, en particulier pour l'Italie (Vaes, 1989) et on la retrouve dans maintes études plus générales (par exemple Demandt, 1989, p. 528-429; Sauer, 1996, p. 75). En Gaule, cette vision est encore renforcée par la Vita, hautement idéalisée, que consacre Sulpice Sévère à son 
héros Martin, dans laquelle il raconte comment le saint détruisit, à la fin $\mathrm{du} \mathrm{IV}^{\mathrm{e}} \mathrm{s}$., des sanctuaires et érigea des églises à leur place (Vie de saint Martin, 13, 9). Dans la première moitié $\mathrm{du} \mathrm{VI}^{\mathrm{e}} \mathrm{s}$., Césaire d'Arles consacre encore un sermon à ce sujet (Sermon, 53). Il s'agit dans ces cas de temples ruraux (voir dans la seconde partie de ce dossier, la contribution de Y. Codou et M.-G. Colin, Gallia, 64, 2007, à paraître). Cependant, J.-P. Caillet a montré dans un article récent que cette pratique n'était pas si généralisée et qu'elle était en tout cas plus tardive que l'on ne le pensait jadis (Caillet, 1996; voir également Dally, 2003 et Sotinel, 2004). L'analyse des villes du midi de la Gaule confirme effectivement ce jugement, car les preuves d'un remplacement d'un temple païen par une église paléochrétienne sont extrêmement rares.

\section{LES TEMPLES PUBLICS}

Nous considérerons d'abord les temples situés sur le forum, souvent liés au culte impérial et donc symboles par excellence du pouvoir antique, ainsi que les autres temples entretenus sur les fonds publics. L'étude de cette question est toutefois compliquée par le fait que peu de temples classiques sont connus, qui auraient fait l'objet d'une fouille permettant de suivre leur évolution durant l'Antiquité tardive.

C'est le cas, par exemple, à Toulouse, où le secteur du forum a été radicalement transformé autour des années 400. À ce moment, le temple du forum (Capitole) a été entièrement et systématiquement détruit, et une partie de son décor a été retaillée sur place. Le secteur n'est pourtant pas abandonné définitivement, car dans le courant du $\mathrm{V}^{\mathrm{e}} \mathrm{s}$., une nouvelle aire de circulation est aménagée, qui peut laisser penser que l'on est toujours sur un domaine public. Enfin, à la fin $d u V^{\mathrm{e}} \mathrm{s}$. ou au début du siècle suivant, plusieurs fosses dépotoirs et des constructions légères témoignent de l'appropriation privée de cet espace, où les ruines du podium restaient cependant encore visibles (Arramond, Boudartchouk, 1996; Boudartchouk, Arramond, 2001). Le souvenir de ce temple ne semble effectivement pas avoir été oublié, puisque sur la partie arrière du podium fut construit un lieu de culte, peut-être dès 567, si l'on se fie à un poème de Venance Fortunat (Carmina, II, 8) qui raconte la consécration d'un "temple » dédié à Saturnin dans les ruines du Capitole; jusqu'au XIX ${ }^{\mathrm{e}}$ s., l'église placée sous le vocable de Saint-Pierre-Saint-Géraud en a gardé le souvenir (fig. 6) (Arramond, Boudartchouk, 1996; Boudartchouk et al., 2001).
Dans d'autres cas, la spoliation des temples a entraîné leur disparition complète dès la fin de l'Antiquité, comme à Saint-Bertrand-de-Comminges où l'on construit, sur l'arase-ment du péribole est du temple du forum, un petit établissement thermal, sans doute à la fin $d u \mathrm{IV}^{\mathrm{e}} \mathrm{s}$ ou au début du $\mathrm{V}^{\mathrm{e}} \mathrm{s}$., bien qu'il soit vrai que la disparition du péribole n'implique pas nécessairement celle du temple (fig. 7) (Badie et al., 1994, p. 62-68; Aupert et al., 2001, p. 97-98; Sablayrolles coord., 2001-2002, p. 39; Bouet, 2003b, p. 567-568). Plus certain est le cas de Nîmes, où les deux temples trouvés lors des fouilles de l'Ancien Théâtre furent complètement détruits dans le courant du $\mathrm{V}^{\mathrm{e}}$ s. (Monteil, 1999, p. 229 et p. 432), à un moment où ce secteur se trouvait selon toute probabilité à l'extérieur d'une nouvelle enceinte, qui ne comprenait plus que le centre-ville (Monteil, 1999, p. 438-440; Heijmans, 2004a, p. 116-117; voir infra, p. 61-63).

La construction d'une nouvelle enceinte laissant à l'extérieur des temples antiques n'a cependant pas forcément entraîné systématiquement la récupération complète de ces derniers, comme le montre le temple de Vésone à Périgueux, qui a certes été spolié de son décor quand l'enceinte réduite a été construite à la fin du $\mathrm{III}^{\mathrm{e}} \mathrm{s}$., mais qui reste toujours debout sur une hauteur considérable (Lauffray, 1990; Girardy-Caillat, 1998, p. 35). On ignore pour quelle raison cet édifice magnifique a échappé à la démolition complète là où d'autres, dans des conditions comparables, ont disparu.

Parfois, les sources médiévales nous permettent de suivre, mieux que l'archéologie, le devenir des temples antiques. Toujours à Nîmes, mais à l'intérieur de l'enceinte tardive, la Maison Carrée doit son exceptionnel état de conservation non pas à sa transformation en église, ce qu'elle n'est devenue qu'au XVII ${ }^{\mathrm{e}}$ s, mais sans doute au fait qu'elle a continué à être utilisée par les pouvoirs publics, bien que ses abords aient été démantelés dès le courant du $\mathrm{V}^{\mathrm{e}} \mathrm{s}$. (Monteil, 1999, p. 192 et p. 432). En effet, les textes montrent qu'au $\mathrm{IX}^{\mathrm{e}}$ s., le temple abritait le Capitole et, bien qu'il soit impossible de savoir depuis quel moment, il n'est pas sans intérêt de rappeler la loi de 407 ordonnant de transformer les temples pour un usage public (C. th., XVI, 10, 19, 1-2).

Quant au «Capitole » de Narbonne, son évolution est en partie parallèle à celle de la Maison Carrée. Signalé aussi bien par Ausone au IV ${ }^{\mathrm{e}}$ s. (Ordo urbium nobilium, XIX, 14-17) que par Sidoine Apollinaire au siècle suivant (Carmina, XXIII, 41), le temple du forum, situé dans la partie nord de la ville, était manifestement encore debout durant 


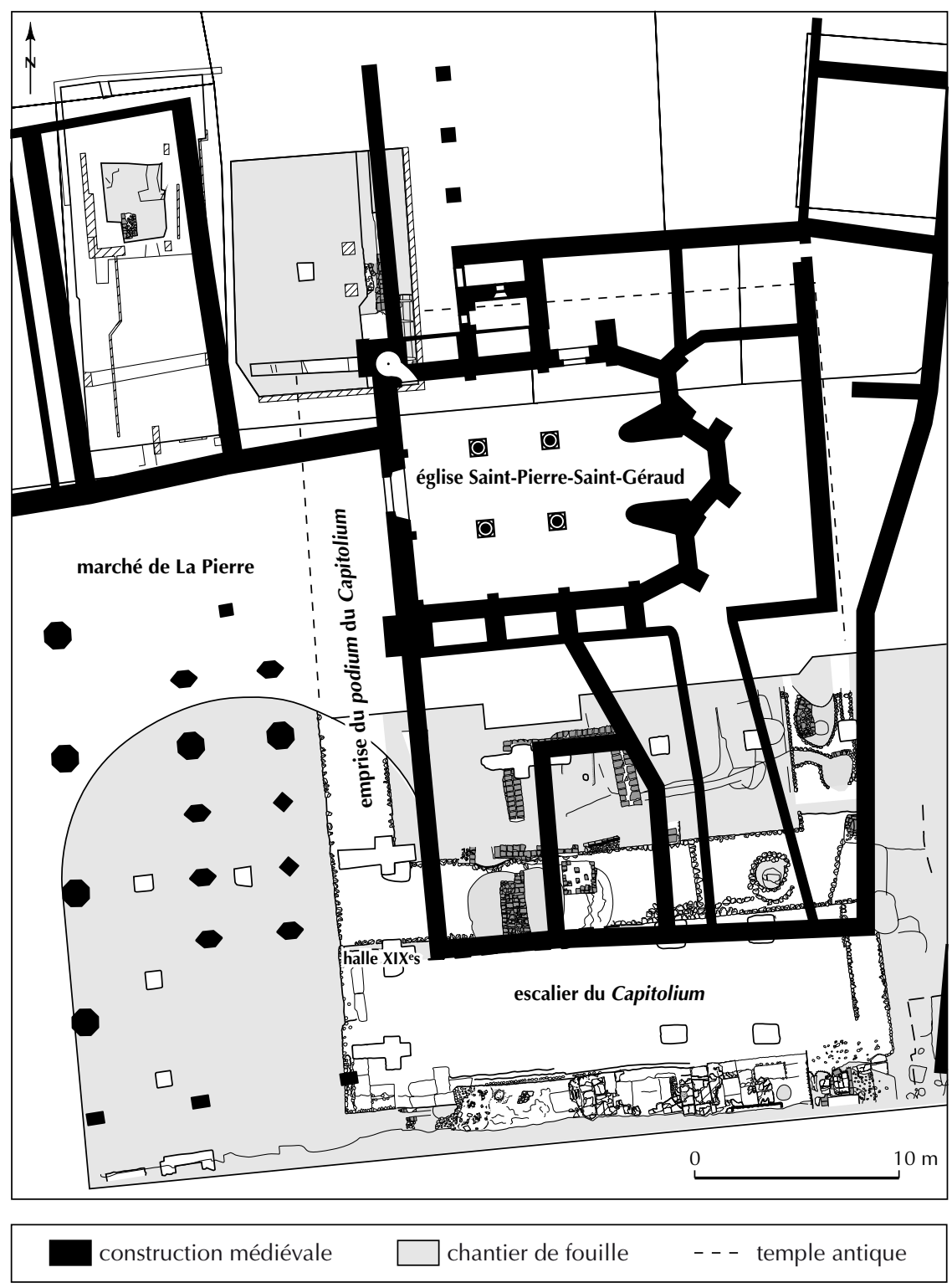

Fig. 6 - Toulouse (Haute-Garonne). Projection de l'église Saint-Pierre-Saint-Géraud sur l'emprise du Capitole (DAO C. Viers, L. Llech et J.-L. Boudartchouk, INRAP).

l'Antiquité tardive. Il est à plusieurs reprises signalé dans les chartes médiévales comme Capitolium. Sans doute déjà en partie ruiné, il a été détruit au $\mathrm{XV}^{\mathrm{e}} \mathrm{s}$. (Gayraud, 1981, p. 268-270).

L'un des rares cas où la transformation d'un temple classique en église chrétienne pendant l'Antiquité est généralement admise est celui du temple d'Auguste et Livie à Vienne, connu jusqu'à l'époque moderne comme NotreDame-de-la-Vie (fig. 8). Cette transformation pourrait avoir eu lieu à la fin $d u V^{\mathrm{e}}$ s. ou au début $\mathrm{du} \mathrm{VI}^{\mathrm{e}} \mathrm{s}$., peut-être sous l'épiscopat d'Avit (494-517) (Pelletier, 1974, p. 59; TCCG, III, 1986b, p. 34). On a également supposé, timidement, pour Toulouse, l'existence d'une église de l'Antiquité tardive ou du haut Moyen Âge, sur l'arasement d'un temple également hypothétique (Cazes, 2001, p. 482-483). Mais aucune des villes où E. Mâle avait postulé le passage d'un temple païen à une église paléochrétienne pour le sud de la Gaule (Mâle, 1950, p. 41-42), n'offre le moindre argument plaidant en faveur de cette hypothèse, uniquement fondée sur des traditions et des légendes. 


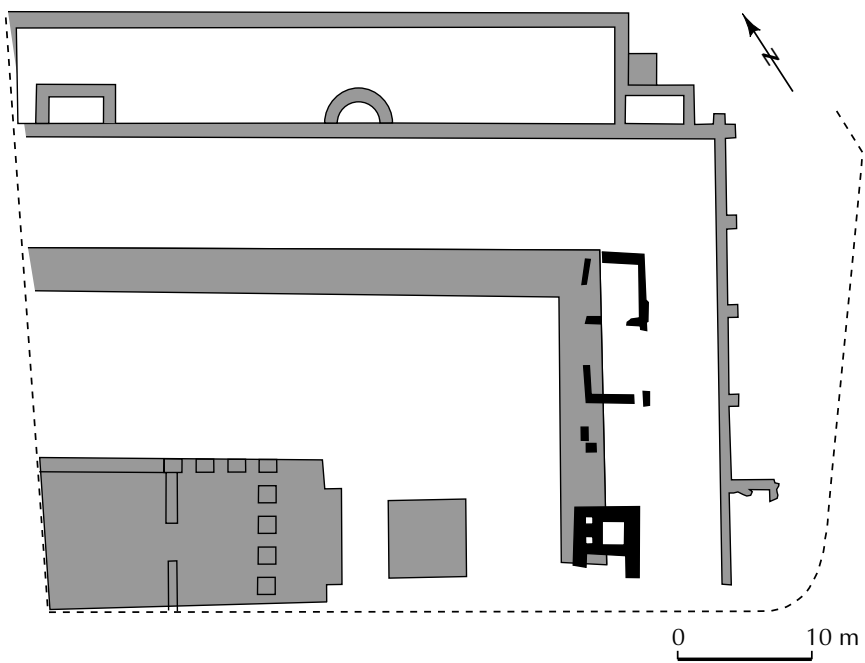

Fig. 7 - Saint-Bertrand-de-Comminges (Haute-Garonne). Plan des thermes tardifs sur l'arasement du péribole est du temple du forum (d'après Badie et al., 1994, fig. 60).

\section{LES AUTRES TEMPLES}

Si les temples publics ont subi une lente dégradation due à l'absence d'entretien, il en est autrement pour des cultes privés, et notamment les mithraea, souvent victimes de la violence des chrétiens, bien que des exemples urbains soient très limités (Sauer, 1996 et 2003). Jérôme cite le cas d'un mithraeum à Rome, détruit vers 376-377 avec ses statues, par le préfet de la Ville, Gracchus, nouvellement converti au christianisme (Lettres, 107, 2) ${ }^{59}$. À Rome, on connaît plusieurs mithraea conservés sous des églises paléochrétiennes, comme sous Sainte-Prisca (Vermaseren, Van Essen, 1965) ou sous Saint-Étienne-le-Rond (LissaCaronna, 1986). Non loin de là, à Ostie, deux temples de Mithra auraient été détruits, et dans l'un d'entre eux fut élevée une église chrétienne (Meiggs, 1973, p. 401).

Pour le midi de la Gaule, les témoignages sont plus rares, car on ne connaît qu'un seul mithraeum dans un chef-lieu de cité, à Bordeaux, sur le site de Parunis (Gallia Informations, 1987-1988/1, p. 105-108; Gaidon-Bunuel, 1991). Ce sanctuaire, situé à l'extérieur de l'enceinte réduite, semble avoir été détruit vers la fin du $\mathrm{IV}^{\mathrm{e}} \mathrm{s}$., mais, comme c'est souvent le cas, il est difficile d'affirmer si cette destruction est due à la violence des chrétiens. Les statues ont en effet été retrouvées basculées dans un niveau de démolition,

59. Voir Prudence, Contre Symmaque, I, v. 561-565. Pour Gracchus, voir PLRE, I, Gracchus 1.

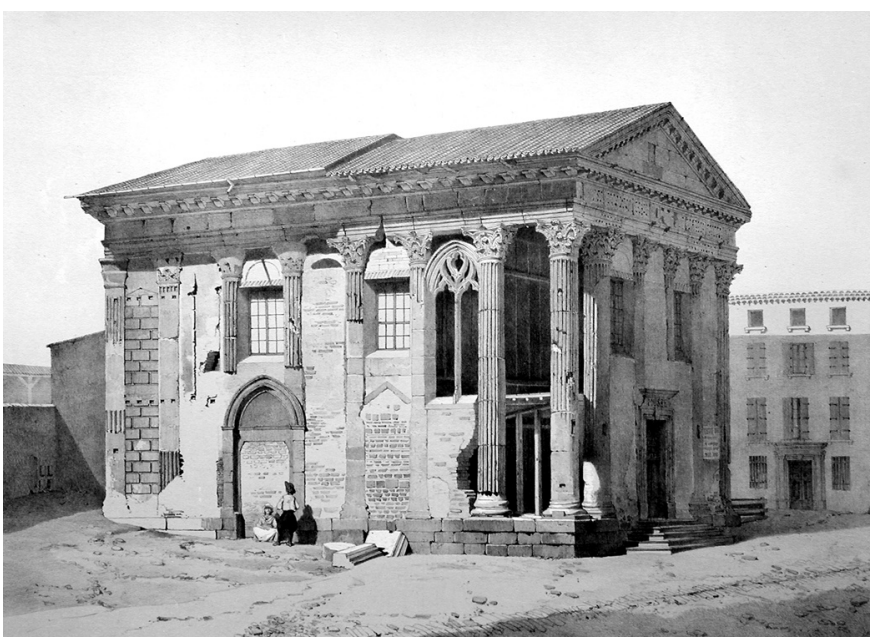

Fig. 8 - Vienne (Isère). Vue ancienne du temple (d'après Debelle, 1978-1979, vol. 2, pl. 79).

mais n'ont pas été mutilées, comme c'est souvent le cas dans les mithraea (Sauer, 2003). Le Mithra taurochtone n'a pas été retrouvé, soit parce qu'il a été totalement détruit et dispersé, soit parce qu'il a été récupéré. C'est peut-être d'un autre mithraeum ou du même sanctuaire qu'est issu le relief réutilisé dans le sol d'un habitat du VI ${ }^{\mathrm{e}} \mathrm{s}$. au sud du parc de stationnement Camille-Jullian (Barraud, Maurin, 1996, p. 52). Au centre de Saint-Bertrand-de-Comminges, a été observé, au nord des thermes du Nord, un sanctuaire atypique, qui a été interprété, grâce à la découverte d'un relief représentant Attis, comme un temple de Cybèle; construit dans le courant du III ${ }^{\mathrm{e}} \mathrm{s}$, il a été détruit à la fin du IV $^{\mathrm{e}}$ s. (Aupert, Turcan, 1995; Sablayrolles coord., 2001-2002, p. 52-54). Quant au temple de Cybèle que l'on a parfois supposé se trouver sous l'église Notre-Dame-la-Major, dans l'angle nord-est de la ville d'Arles, sur la foi d'une stèle dédiée à la Bonne Déesse trouvée dans une citerne devant l'église (Constans, 1921, p. 350-351), elle se heurte à la fois au fait que la stèle ne vient pas nécessairement de cet endroit et que rien ne dit que cette église remonte à l'Antiquité tardive.

\section{LE FORUM, LES AUTRES PLACES PUBLIQUES ET LA VOIRIE}

Nous avons déjà abordé en partie la question du forum, avec l'étude de la survie des temples publics. Il semble bien qu'à quelques exceptions près, comme à Glanum, où l'on peut supposer que le forum a été abandonné, avec le reste de la ville, à la fin du $\mathrm{III}^{\mathrm{e}}$ s. (Salviat, 1990, p. 22-23), les fora des 
villes de sud-est de la Gaule ont gardé leur fonction politique et économique pendant tout le $\mathrm{IV}^{\mathrm{e}} \mathrm{s}$. À Arles, une fouille sous l'Hôtel de Ville a montré qu'à cet endroit, le stylobate avait été démonté partiellement et que des maisons ou des boutiques ont été installées directement sur le dallage antique, sans doute au début du $\mathrm{V}^{\mathrm{e}} \mathrm{s}$. (fig. 9) (Sintès, 1994, p. 182; Heijmans, 2004a, p. 367-369). Malheureusement, la superficie réduite de la fouille interdit d'étendre ces observations à l'ensemble du forum, qui, s'il faut en croire Sidoine Apollinaire, était encore le centre de la vie dans les années 460 (Lettres, I, 11).

À Aix, c'est encore plus tard, sans doute pas avant les années 500, que l'on installe sur la partie nord du forum le groupe épiscopal, avec le baptistère qui repose directement sur le dallage antique, tandis que la cathédrale primitive se trouvait peut-être à l'emplacement de la basilique civile, au nord du forum (fig. 10) (Guyon et al., 1998, p. 100-101). Cette installation tardive, plus d'un siècle après l'attestation du premier évêque aixois, peut surprendre et suppose l'existence ailleurs d'une cathédrale, sans doute à Notre-Dame-de-laSeds, dans la partie ouest de la ville, comme le veut la tradition (TCCG, II, 1986a, p. 26). Le fait que, vers 500, l'évêque d'Aix, peut-être Basilius, pouvait s'approprier le centre civique pour construire sa cathédrale, montre bien qu'à ce moment, quand la Provence était sous le pouvoir des Wisigoths et qu'Aix n'avait plus de rôle politique important, le forum pouvait facilement être amputé d'une large partie de sa superficie.

La situation était différente à Fréjus, où la cathédrale est installée au début $d u \mathrm{~V}^{\mathrm{e}} \mathrm{s}$. immédiatement à l'ouest de ce que les analyses pertinentes de Lucien Rivet et ses collègues ont permis d'identifier comme un centre monumental dans lequel ils reconnaissent, avec une grande probabilité, le forum antique (Rivet et al., 2000, p. 392-397). Or, bien que seule une faible partie en ait été fouillée, il paraît bien que cette place n'a été remblayée que tard dans le $\mathrm{VI}^{\mathrm{e}} \mathrm{s}$. et que, comme à Arles, le groupe épiscopal et le centre politique ont ici coexisté.

Pour les autres villes provençales, les renseignements sont moins explicites. Pour les cités vauclusiennes, les études en cours de D. Carru dans le cadre du projet collectif de recherche "Topographie urbaine de Gaule méridionale ", lui ont permis d'étudier le forum d'Avignon et sa possible mise en défense durant l'Antiquité tardive, tandis qu'à Cavaillon, il n'est pas impossible que la cathédrale ait été construite au-dessus ou à proximité du forum (Carru, à paraître). On retrouve sans doute un cas de figure analogue à Carpentras, où l'arc de triomphe antique, probablement une entrée au forum, a servi de porche d'accès dans le mur

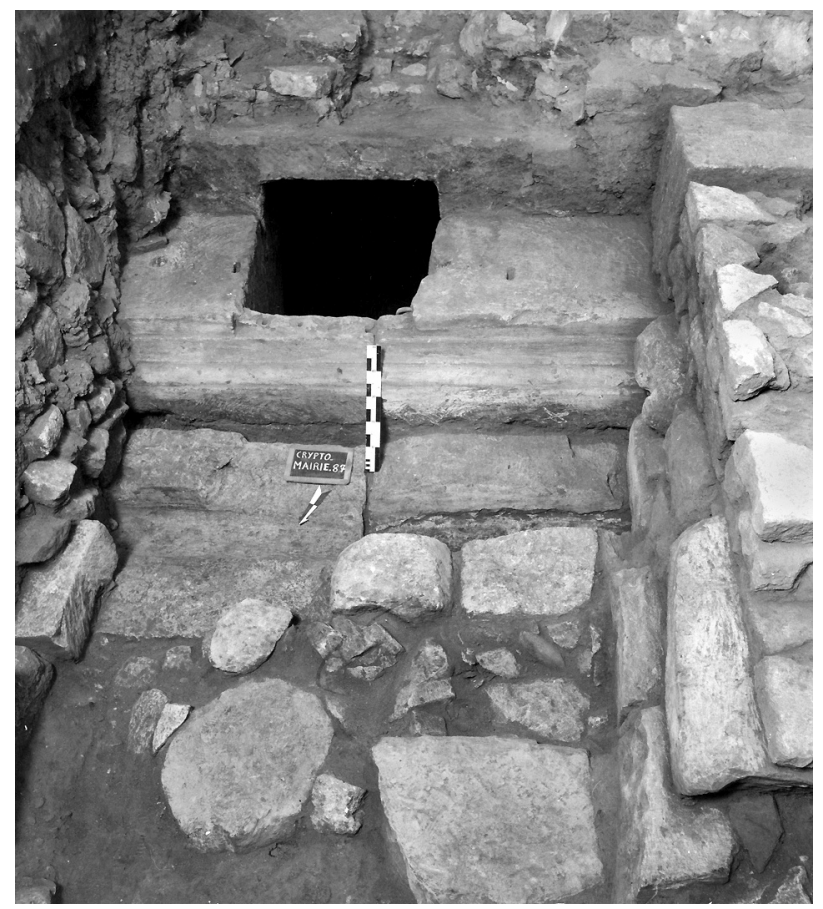

Fig. 9 - Arles (Bouches-du-Rhône). Vue des vestiges antiques sur le forum (cliché M. Lacanaud, musée de l'Arles et de la Provence antiques).

latéral nord de la cathédrale romane (id., ibid.) et peut-être également à Apt (Tallah, 2004, p. 65). Dans ces derniers cas, la filiation entre forum et cathédrale est cependant plus supposée que démontrée. Pour les autres villes de Provence enfin, on ignore jusqu'à l'emplacement du forum dans la cité.

Quant au Languedoc, la situation n'est guère meilleure. On connaît bien sûr la localisation et l'emprise du forum de Nîmes, grâce à la présence du temple dit la Maison Carrée et aux fouilles récentes aux alentours, mais l'état des vestiges durant l'Antiquité tardive demeure obscur. Il semble cependant que les premières spoliations aient eu lieu dès le courant du $\mathrm{V}^{\mathrm{e}} \mathrm{s}$. (Monteil, 1999, p. 432) et c'est sans doute à la même période qu'à Toulouse le secteur du forum fût transformé, comme nous l'avons mentionné plus haut (Arramond, Boudartchouk, 1996; Boudartchouk, Arramond, 2001; voir supra, p. 28).

En Aquitaine, la situation est assez différente car, dans plusieurs cas, le centre civique a été laissé à l'extérieur des enceintes créées pendant l'Antiquité tardive, entraînant quasi systématiquement l'abandon des bâtiments administratifs (Aupert, Sablayrolles, 1992, p. 291; Maurin, 1992, p. 369). Ainsi, pour les enceintes du premier groupe qu'a distinguées L. Maurin (1992, p. 369-373), construites à la fin $d u \mathrm{III}^{\mathrm{e}} \mathrm{s}$. ou au début du siècle suivant, le forum se 

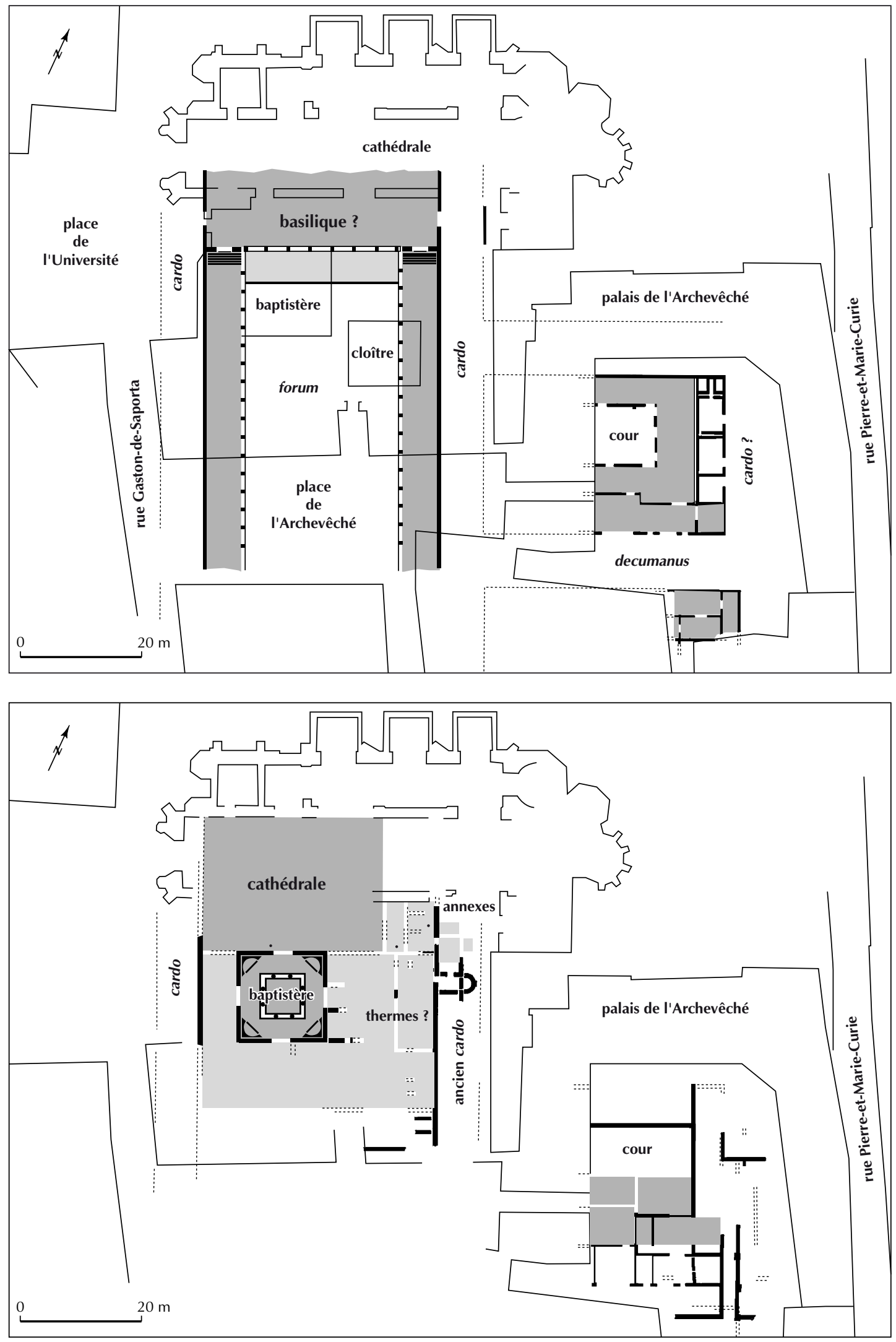

Fig. 10 - Aix-en-Provence (Bouches-du-Rhône). Plans du forum antique et du groupe épiscopal (d'après Duval dir., 1995, p. 113). 
trouve désormais extra muros à Périgueux, comme on vient de le voir à propos du temple de Vésone, et probablement aussi à Poitiers, bien que l'emplacement exact du forum reste discuté. À Saintes, la réutilisation dans l'enceinte des éléments architecturaux provenant de l'ancien centre civique témoigne de sa désaffectation tandis qu'à Bordeaux, les Piliers de Tutelle, dans lesquels on a voulu voir un sanctuaire ou un second forum, ont été laissés à l'extérieur de la ville (fig. 11) (Barraud, Maurin, 1996). Si leur conservation jusqu'au XVII ${ }^{\mathrm{e}} \mathrm{s}$. montre que ce monument n'a pas été victime d'un pillage systématique, on n'en avait manifestement plus l'utilité pour la vie civile. Ainsi, la seule ville où le centre public est resté à l'intérieur des nouveaux murs des $\mathrm{III}^{\mathrm{e}}-\mathrm{IV}^{\mathrm{e}}$ s. semble être Bourges, peut-être plutôt à cause des contraintes du relief qu'à son rôle de capitale de la province d'Aquitaine Première. Il faut peut-être rapprocher de Bourges le cas de Rodez, où le tracé et la date de l'enceinte demeurent inconnus, mais dont on est sûr que le centre civique est intégré dans l'enceinte réduite. Or, la fouille du site des Jacobins, en plein cœur de la ville, a montré les avatars du forum de la cité ruthénoise durant l'Antiquité tardive, après sa destruction à l'extrême fin du III ${ }^{\mathrm{e}} \mathrm{s}$. Dans la première moitié $d u I^{e} \mathrm{~s}$., un foyer est aménagé au sein d'une des boutiques sud, remplacé dans un deuxième temps (seconde moitié du IV ${ }^{\mathrm{e}}$ s.-début du $\mathrm{V}^{\mathrm{e}} \mathrm{s}$.) par un nouveau foyer, après le dédoublement de la surface de la boutique. Cependant, comme le notent à juste titre les fouilleurs, les espaces publics n'ont forcément pas été touchés par cette installation. À partir du V $\mathrm{V}^{\mathrm{e}}$ s. en revanche, le site est définitivement abandonné (Catalo et al., 1999).

Dans le deuxième groupe d'enceintes, la question se pose différemment, et cela pour plusieurs raisons : d'abord, ces enceintes, qui se rencontrent essentiellement au sud de la Garonne, à une exception près, sont des fortifications de faible superficie, aménagées plus tardivement, probablement au début $\mathrm{du} \mathrm{V}^{\mathrm{e}} \mathrm{s}$.; d'autre part, elles sont construites

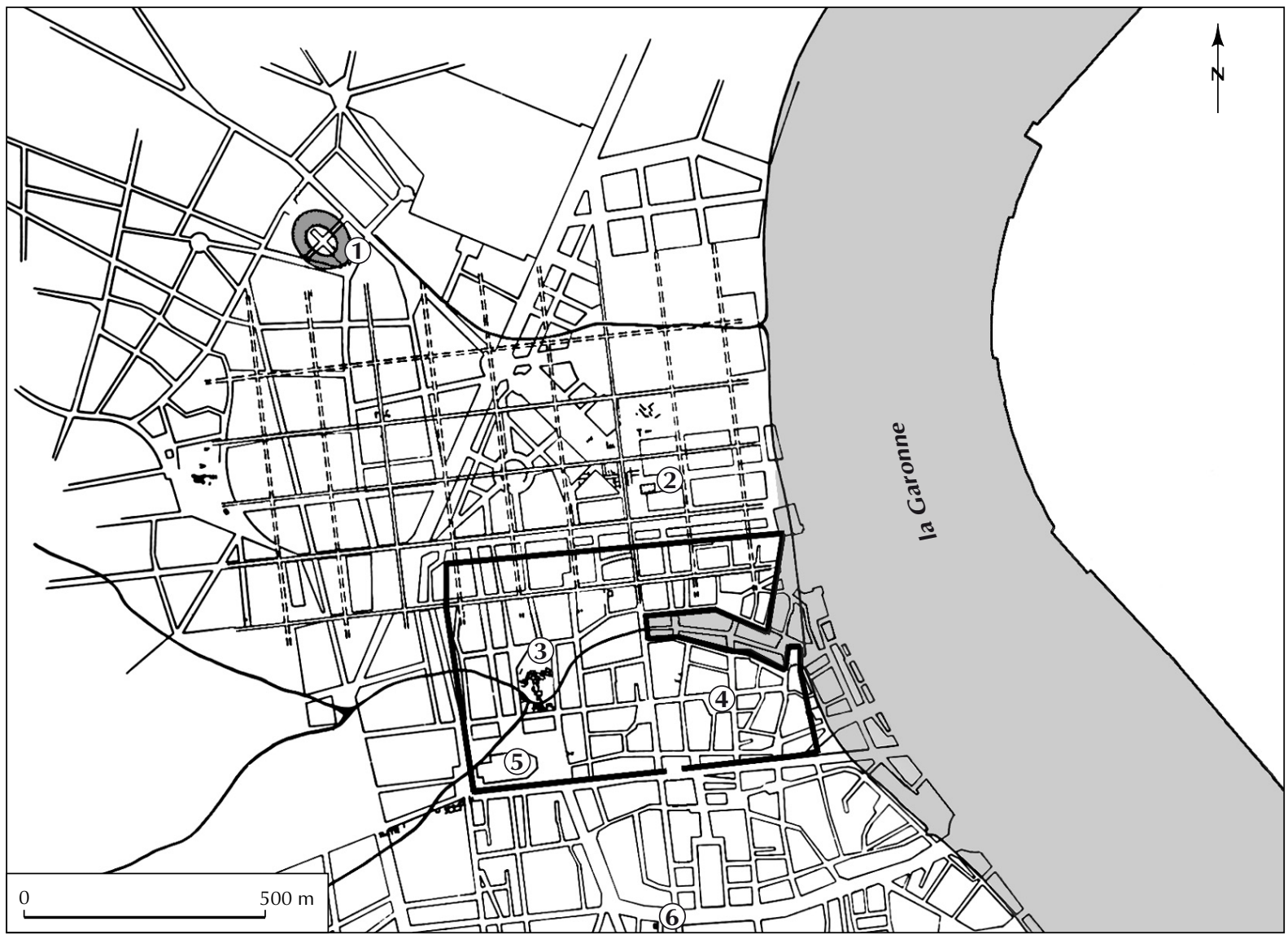

Fig. 11 - Bordeaux (Gironde). Plan de la ville pendant l'Antiquité tardive : 1, amphitéâtre (Palais Gallien); 2, les Piliers de Tutelle ; 3, îlot Saint-Christoly ; 4, place Camille-Jullian ; 5, cathédrale ; 6, Parunis (d'après Barraud, Gaidon, 1992, p. 45, fig. 19). 
sur des hauteurs dominant la ville du Haut-Empire et il y a donc eu un véritable déplacement du centre urbain. De ce fait, il n'y a, à priori, pas de raisons de supposer un abandon de la ville basse et, avec elle, du centre civique avant le début $\mathrm{du} \mathrm{V}^{\mathrm{e}} \mathrm{s}$., et l'occupation de la ville basse peut même coexister avec la ville haute, qui a surtout une fonction de citadelle. Enfin, plusieurs de ces villes n'ont pas joué un rôle politique important durant le Haut-Empire et ne sont devenus chefs-lieux de cité que pendant l'Antiquité tardive; il s'agit d'Aire-sur-l'Adour, Lescar, Tarbes/Saint-Lézer, la cité des Boiates, Saint-Lizier et Oloron-Sainte-Marie (Esmonde Cleary, 2004, p. 184-186; Maurin, 2004). Comme d'autres agglomérations secondaires de l'Aquitaine, elles n'avaient donc pas de centre civique proprement dit, ou en tout cas beaucoup plus modeste que dans les cités (Aupert, Sablayrolles, 1992, p. 289-290). Malheureusement, dans la majorité des cas, l'absence de fouille empêche de savoir en détail comment était organisée la ville basse. C'est le cas de Bazas, Saint-Lézer ou Saint-Lizier, où l'on ignore même si elle a ou non existé (TCCG, XIII, 2004, p. 116-120). C'est également le cas d'Auch, Lectoure ou Lescar, où l'occupation de la ville basse aux $\mathrm{IV}^{\mathrm{e}}$ et $\mathrm{V}^{\mathrm{e}} \mathrm{s}$. est bien attestée, mais où l'on ignore tout du centre civique du Haut-Empire (TCCG, XIII, 2004, p. 20-22, p. 44-46 et p. 102-104). De fait, la seule cité dont on peut suivre l'évolution de la ville basse durant l'Antiquité tardive est Saint-Bertrand-de-Comminges, grâce aux fouilles programmées dans cette ville depuis les années 1990 (Sablayrolles coord., 2001-2002, p. 75; TCCG, XIII, 2004, p. 51-81). Or, il apparaît que les monuments publics, comme la voirie, ont encore été maintenus en état, voire restaurés durant le $\mathrm{IV}^{\mathrm{e}} \mathrm{s}$. et que ce n'est qu'à la fin $d u \mathrm{IV}^{\mathrm{e}} \mathrm{s}$. ou au début $\mathrm{du} \mathrm{V}^{\mathrm{e}} \mathrm{s}$., au moment où est construite l'enceinte de la ville haute, que ces monuments sont spoliés.

Comme on vient de le voir, ce qui vaut pour le forum vaut aussi en grande partie pour le reste du centre monumental et pour la voirie, avec parfois un léger décalage chronologique. C'est du moins ce qui ressort de l'étude du cas d'Arles, où l'on a pu constater à plusieurs reprises que les rues ont été obstruées (fig. 12), entièrement ou en partie, vers les années 420-430, soit une génération après l'occupation des monuments publics, notamment les édifices de spectacle sur lesquels nous reviendrons, qui se situe autour des années 400 (Sintès, 1994; Heijmans, 2004a, p. 353-379; voir infra, p. 38-39). Cette appropriation des espaces publics pour des fins privées a également été observée ailleurs, comme à Apt, où un pressoir est construit sur l'emprise du

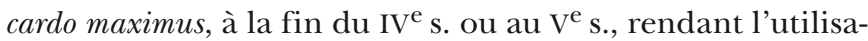
tion de ce dernier difficile (Tallah, 2004, p. 101). Plusieurs

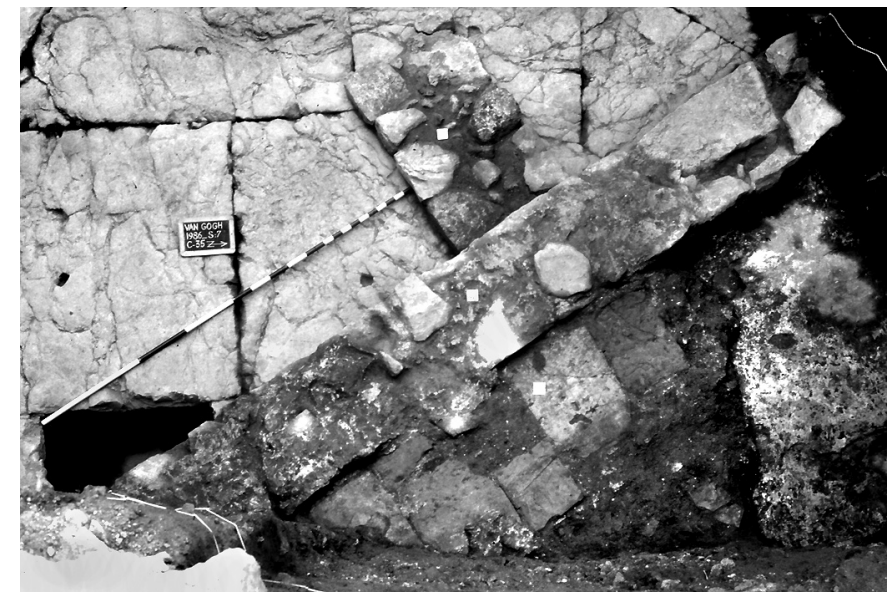

Fig. 12 - Arles (Bouches-du-Rhône). Vue des maisons sur le dallage de la place de l'hôpital Van-Gogh (cliché M. Lacanaud, musée de l'Arles et de la Provence antiques).

fouilles toulousaines montrent cependant le maintien, voire l'amélioration de la voirie durant les $\mathrm{V}^{\mathrm{e}}$ et $\mathrm{VI}^{\mathrm{e}} \mathrm{s}$. dans des cas particuliers, ce qui n'empêche pas des empiètements sur les trottoirs et les bas-côtés (Arramond et al., 2001; Filippo, 2001a et b). Il s'agit cependant d'un phénomène qui n'est pas propre à l'Antiquité tardive, car l'empiètement sur le domaine public est observé dès avant la fin du $\mathrm{III}^{\mathrm{e}} \mathrm{s}$. (Desbordes, Loustaud, 1992, p. 246).

\section{LES THERMES PUBLICS}

Un autre phénomène typique des villes romaines et bien diffusé dans le midi de la Gaule, le devenir des thermes publics durant l'Antiquité tardive, a moins retenu l'attention des historiens. Les sources tardo-antiques parlent pourtant souvent des thermes, dont l'usage était proscrit par les Pères de l'Église, comme Jérôme, qui estimait que le lavage par le Christ (donc le baptême) suffisait pour un chrétien (Lettres, 14, 10). En pratique, la position de l'Église était plus tolérante, en particulier envers les thermes curatifs et plusieurs textes montrent l'existence de thermes liés aux groupes épiscopaux, destinés aussi bien au clergé qu'aux pauvres (Ward-Perkins, 1984, p. 135-146; Yegül, 1992, p. 315-320). Le maintien des thermes était également un souci des pouvoirs publics, comme en témoigne une loi promulguée en 395, destinant, pour éviter que les villes les plus splendides ne tombent en ruines, un tiers des revenus fonciers de l'État à la réparation des monuments publics et au chauffage des thermes (C. th., XV, 1, 32). Pour l'Italie, B. Ward-Perkins a observé que la seule catégorie d'édifices 
publics pour laquelle la réparation est régulièrement documentée par l'épigraphie est bien celle des thermes (Ward-Perkins, 1984, p. 31; Yegül, 1992, p. 321-323 et pour l'Espagne, Jiménez Sanchez, Sales Carbonell, 2004).

Grâce aux études récentes de A. Bouet, nous disposons désormais d'un survol de l'évolution des thermes de la Gaule du Sud (Bouet, 2003a et b). On peut constater un abandon important des établissements thermaux au cours du III ${ }^{\mathrm{e}}$ s., tant en ville qu'à la campagne. Au IV ${ }^{\mathrm{e}}$ s., on assiste certes à une nouvelle vague de construction, mais qui ne compense pas pour autant l'abandon d'autres installations thermales. $\mathrm{Au} \mathrm{V}^{\mathrm{e}} \mathrm{s}$. enfin, la construction de nouveaux thermes se limite essentiellement aux installations liées à des groupes épiscopaux.

Si nous concentrons notre attention sur les villes, le tableau se précise. En ce qui concerne les thermes urbains construits au Haut-Empire, la majorité est abandonnée à la fin du III $^{\mathrm{e}} \mathrm{s}$. C'est le cas pour plusieurs établissements publics situés dans les quartiers suburbains des villes, qui ont subi le sort général de ces quartiers. On peut ainsi signaler les thermes des Lutteurs, à Saint-Romain-en-Gal (Bouet, 2003a, no 157), ou, dans le même quartier, les Petits Thermes du Nord-Est (id., ibid., no 158). À Vaison, les thermes du Nord sont abandonnés vers 285 (id., ibid., $\mathrm{n}^{\mathrm{o}}$ 195). Accolés à l'enceinte, mais toujours extra muros, les thermes de l'Esplanade, à Arles (id., ibid., $\mathrm{n}^{\circ} 27$ ), sont délaissés vers 260-270 et c'est sans doute vers la même période qu'il faut situer l'abandon des thermes du Clos Saint-Antoine à Fréjus, au pied de la butte du même nom (Rivet et al., 2000, p. 284; Bouet, 2003a, no 60), tandis qu'à Bordeaux, c'est la construction même de l'enceinte tardive qui est à l'origine de la destruction des thermes de la rue des Frères-Bonie (Bouet, 2003b, p. 553-554). L'abandon précoce de ces installations publiques ne se limite cependant pas à la périphérie des villes; à Nîmes, en plein cour de la cité, les thermes de la ZAC des Halles sont partiellement désaffectés dès la fin $d u \mathrm{II}^{\mathrm{e}} \mathrm{s}$. ou le début du $\mathrm{III}^{\mathrm{e}} \mathrm{s}$., et l'abandon total intervient au milieu du III $^{\mathrm{e}}$ s. (Monteil, 1999, p. 430-431; Bouet, 2003a, no 114).

En revanche, les témoignages de la continuité du fonctionnement des thermes urbains entre le $\mathrm{III}^{\mathrm{e}} \mathrm{s}$. et le $\mathrm{IV}^{\mathrm{e}} \mathrm{s}$. sont extrêmement peu nombreux et de surcroît parfois discutables. Ainsi, l'utilisation au $\mathrm{IV}^{\mathrm{e}} \mathrm{s}$. des thermes dits la Basilique, à Vaison, est uniquement suggérée par la découverte d'une lampe du $\mathrm{IV}^{\mathrm{e}} \mathrm{s}$. dans un égout, ce qui n'empêche pas, comme le souligne A. Bouet, que l'abandon du complexe a pu être antérieur (Bouet, 2003a, no 193). À Toulouse, le même cas de figure se retrouve aux thermes de la rue du Languedoc, où la présence de céramique estampée dans un égout est, pour le fouilleur, un argument pour dater cet ensemble de l'Antiquité tardive (Baccrabère, 2001), tandis que A. Bouet y voit un argument pour dater du IV ${ }^{\mathrm{e}}$ s. l'abandon de ces thermes (Bouet, 2003a, no 84). Également incertain est le maintien des thermes de Cahors, qui ne semblent désertés qu'au IV ${ }^{\mathrm{e}} \mathrm{s}$., voire au V $\mathrm{V}^{\mathrm{e}} \mathrm{s}$., sans que les arguments soient vraiment convaincants (Labrousse, 1963, p. 222-223 ; Bouet, 2003b, p. 558). Cependant, à SaintBertrand-de-Comminges, les thermes du forum ont été entretenus et restaurés au IV ${ }^{\mathrm{e}}$ s., avant leur abandon vers 400, puis leur récupération systématique (Aupert et al., 2001, p. 9497; Bouet, 2003b, p. 574). À Marseille, les thermes de la place Villeneuve-Bargemont, sur la rive nord du Vieux-Port, restent en usage, après des remaniements, jusqu'à la fin du $\mathrm{IV}^{\mathrm{e}} \mathrm{s}$. (Hesnard, 1999). Ces derniers cas semblent toutefois former une exception et les seuls thermes du Haut-Empire qui ont encore été fréquentés au $\mathrm{IV}^{\mathrm{e}} \mathrm{s}$., d'après les découvertes monétaires, sont les établissements curatifs (Bouet, 2003a, p. 338).

Si donc la grande majorité des thermes du Haut-Empire ne sont plus utilisés au $\mathrm{IV}^{\mathrm{e}} \mathrm{s}$., on en a construit d'autres, de taille souvent plus modeste, la grande exception étant les thermes de Constantin à Arles, qui s'explique par le rôle politique de cette ville au début du $\mathrm{IV}^{\mathrm{e}} \mathrm{s}$. (fig. 13) (Heijmans, 2004a). Les autres thermes publics construits au IV $^{\mathrm{e}}$ s. dans le midi de la Gaule, comme ailleurs (Yegül, 1992, p. 321; Liebeschuetz, 2001, p. 30), sont effectivement bien plus modestes et très rares; quelquefois, ils sont aménagés dans des quartiers qui n'avaient pas été urbanisés auparavant. C'est le cas des thermes de la place Saint-Étienne à Toulouse, où un premier édifice thermal est construit au début $d u V^{\mathrm{e}} \mathrm{s}$., puis détruit vers le milieu du siècle pour être remplacé par un édifice plus important, détruit à son tour au plus tard au début du VI ${ }^{\mathrm{e}} \mathrm{s}$. (Filippo, 2001a et b; Bouet, 2003a, n $\left.{ }^{\text {os }} 182-183\right)$. L’aménagement de ce quartier périphérique, situé près de la limite orientale de la ville, est peut-être lié à la construction du groupe épiscopal (Cazes, 2001). Un autre exemple est donné par le cas de Saint-Rémyde-Provence, où, au centre du village actuel, à quelques kilomètres au nord du site antique de Glanum, abandonné semble-t-il vers la fin du III $^{\mathrm{e}}$ s., est construite une nouvelle agglomération, qui n'est certes jamais devenue chef-lieu de cité ou siège d'un évêché, mais où l'on a construit un ensemble thermal, comprenant, d'après l'analyse de A. Bouet, plusieurs établissements publics et privés encore conservés actuellement dans l'hôtel de Sade (Bouet, 2003a, $\left.\mathrm{n}^{\text {os }} 151-154\right)$. 


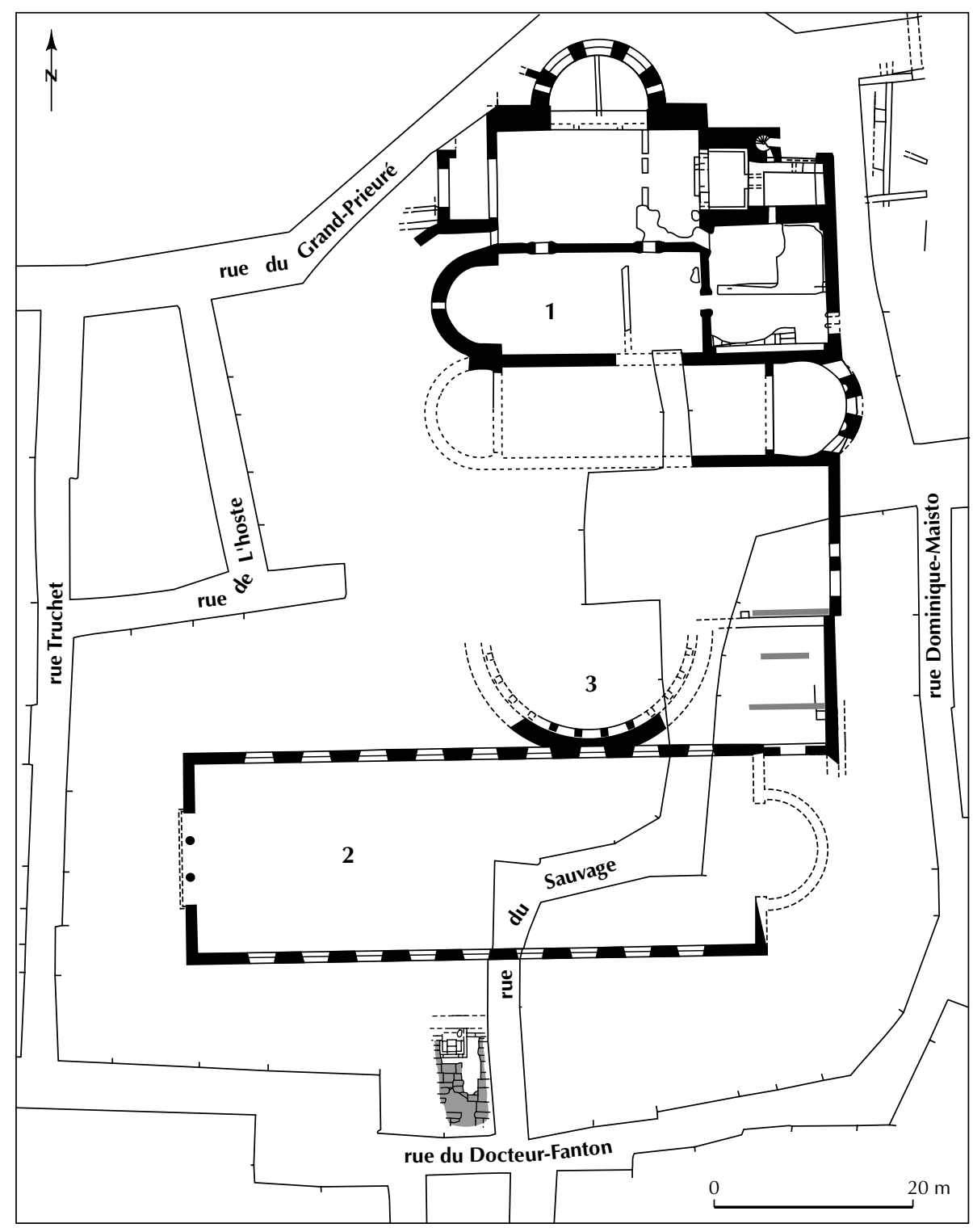

Fig. 13 - Arles (Bouches-du-Rhône). Plan du centre monumental :

1, thermes de Constantin ; 2, basilique civique; 3, extension des thermes (dessin J. Brémond et M. Heijmans, CNRS).

On voit d'autre part, cas également très rare, l'installation de thermes dans des édifices publics abandonnés. Le meilleur exemple est sans doute celui, déjà cité plus haut, des thermes installés à la fin $\mathrm{du} \mathrm{IV}^{\mathrm{e}} \mathrm{s}$. au plus tôt, sans doute après l'abandon des thermes du forum, sur la branche est du péribole du temple du forum à Saint-Bertrand-deComminges. Cette construction, de médiocre qualité, n'a cependant pas servi longtemps et son abandon est intervenu avant la fin $\mathrm{du} \mathrm{V}^{\mathrm{e}}$ s. (Badie et al., 1994, p. 62-68; Aupert et al., 2001, p. 97-98; Sablayrolles coord., 2001-2002, p. 39 ; Bouet, 2003b, p. 567-568). Les thermes installés à la fin du $\mathrm{IV}^{\mathrm{e}} \mathrm{s}$. sur les restes d'un monument public, faisant probablement partie du centre civique, à Apt, sont sans doute plutôt privés que publics (Bouet, 2003a, no 24; Tallah, 2004, p. 100).

Le dernier cas de figure évoqué, celui des thermes directement liés à l'Église et son clergé, est relativement peu attesté. On peut cependant noter l'exemple d'Aix-enProvence, où des thermes de bien modestes dimensions ont été construits près du baptistère vers la fin $\mathrm{du} \mathrm{V}^{\mathrm{e}} \mathrm{s}$., avant d'être abandonnés peu de temps après (Bouet, 
2003a, no 10). On observe le même phénomène à Cimiez, où des petits thermes ont été construits au nord du groupe épiscopal, mais dans un cas bien particulier. En effet, la cathédrale de Cimiez est elle-même aménagée dès le $\mathrm{V}^{\mathrm{e}} \mathrm{s}$. dans d'anciens thermes, dits thermes de l'Ouest, qui étaient probablement déjà en assez mauvais état, car la plupart des murs ont dû être reconstruits au moment de la transformation de l'édifice pour le culte chrétien (fig. 14) (Benoit, 1977, p. 137-153; Février, 1995). Cette cathédrale est installée dans un quartier marqué par la présence d'autres établissements balnéaires du Haut-Empire, qui abritaient à partir des $I^{\mathrm{e}}-\mathrm{V}^{\mathrm{e}} \mathrm{s}$. des habitations grossièrement bâties (Benoit, 1977, p. 159-160; Ardisson, 2004, p. 251-254).

Il s'agit là de l'exemple le plus connu d'une réutilisation chrétienne d'un édifice balnéaire, mais ce n'est pas le seul. C'est sans doute aussi le cas du baptistère de Riez, dont on a supposé, d'après des observations déjà anciennes, qu'il était établi au-dessus des thermes publics (Barruol, 1995, p. 89), ce que semblent confirmer les recherches en cours sur le groupe épiscopal conduites sous la direction de P. Borgard et de C. Michel d'Annoville. La même transformation est attestée, par les sources littéraires, à Amélie-les-Bains, mais pas avant le VIII ${ }^{\mathrm{e}}$ s. (Durliat, 1986, p. 24). C'est également sur des indices indirects que P. Aupert a supposé l'existence d'un lieu de culte chrétien dans les thermes du forum à Saint-Bertrand-de-Comminges (Aupert et al., 2001, p. 99).

Souvent, on observe des traces d'habitations, comme on vient de le voir pour les thermes du Nord et de l'Est à Cimiez ou pour les thermes de la place Villeneuve-Bargemont à Marseille (Hesnard, 1999). Parfois, ces traces d'habitations sont liées, comme c'est le cas pour les thermes de l'Esplanade à Arles, à la récupération systématique des matériaux. La même solution a été proposée pour expliquer l'état de récupération des thermes de Cahors (Labrousse, 1963, p. 222-223). Enfin, cas plus rare, dû à leur emplacement à la périphérie, les ruines de certains thermes ont laissé la place à des tombes, comme au Clos Saint-Antoine à Fréjus (Rivet et al., 2000, p. 284), aux thermes de Saint-Saloine à Saintes (Bouet, 2003b, p. 578-279) et aux thermes du forum à SaintBertrand-de-Comminges (Aupert et al., 2001, p. 99).

\section{LES AQUEDUCS}

La question des thermes est directement liée à celle de l'adduction d'eau. Là aussi, les données archéologiques sont très minces pour le midi de la Gaule. Après une première phase d'interruption dans le courant du III ${ }^{\mathrm{e}}$ s., marquée par des traces de dégradation, l'aqueduc de Nîmes, qui

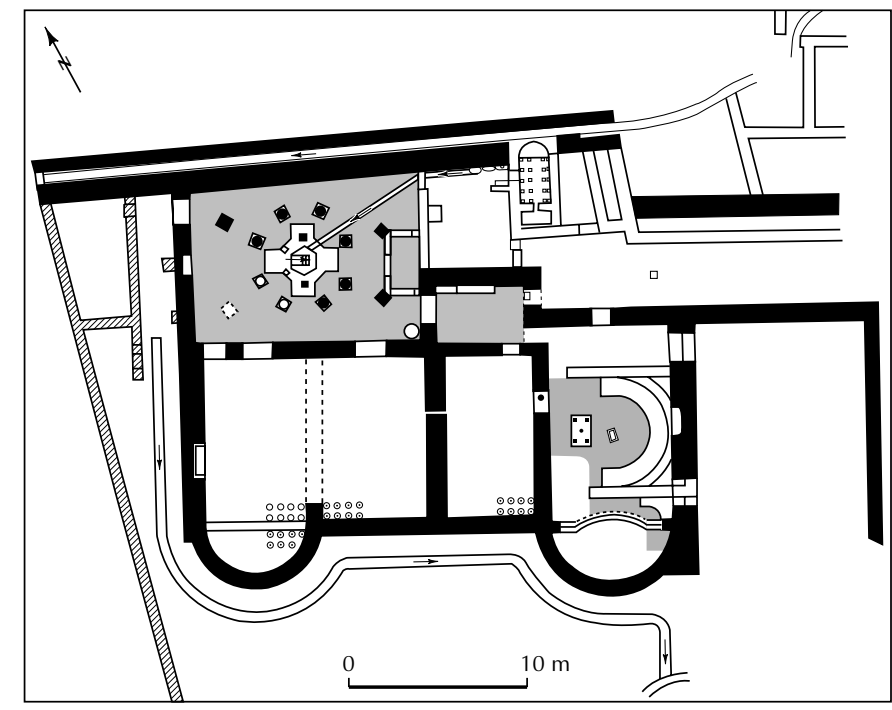

Fig. 14 - Cimiez (Alpes-Maritimes). Groupe épiscopal construit dans les thermes (d'après Duval dir., 1995, p. 104).

est pourtant une ville très mal connue durant l'Antiquité tardive, a été réhabilité dans le courant du $\mathrm{IV}^{\mathrm{e}} \mathrm{s}$. ou au début $\mathrm{du} \mathrm{V}^{\mathrm{e}} \mathrm{s}$, , avant son abandon définitif au début $\mathrm{du} \mathrm{VI}^{\mathrm{e}} \mathrm{s}$. En revanche, pour Fréjus, les auteurs d'une étude récente estiment qu'après la réfection de plusieurs arches dans la seconde moitié du III ${ }^{\mathrm{e}}$ s., l'aqueduc a définitivement cessé de fonctionner au début $\mathrm{du} \mathrm{IV}^{\mathrm{e}} \mathrm{s}$., en partie à cause de l'assèchement de la source de Neisson (Gébara et al., 2002, p. 240 et p. 301-302). On a également supposé une reconstruction de l'aqueduc d'Arles au IV ${ }^{\mathrm{e}}$ s. (Leveau, 1995, p. 128-130 et p. 134-135). À Narbonne, une inscription incomplète et non datée, mais datant sans doute du début de la première moitié du V $\mathrm{V}^{\mathrm{s}}$. (CIL, XII, 4355), mentionne, entre autres, la restauration de l'aqueduc, sur ordre d'un préfet du prétoire des Gaules anonyme, peut-être Marcellus, qui est également intervenu en 441 pour la restauration de la cathédrale de la ville par l'évêque Rusticus (CIL, XII, 5336).

Pour le sud-ouest, on est encore moins bien renseigné; on peut toutefois supposer que la construction des enceintes à la fin du $\operatorname{III}^{\mathrm{e}} \mathrm{s}$. a dû compromettre le maintien des aqueducs, s'ils n'étaient pas déjà abandonnés auparavant. Pour Cahors, ville où une enceinte tardive n'est pas attestée, M. Labrousse avait supposé que la rupture de l'aqueduc était à l'origine de l'abandon des thermes de la ville, au IV ou V ${ }^{\mathrm{e}}$ s. (Labrousse, 1963, p. 223). Dans ce cas, nous avons aussi un précieux témoignage littéraire, qui nous dit que l'évêque Desidirius (635-650), également connu pour avoir construit l'enceinte de la ville, aurait essayé de réparer 
l'alimentation en eau de la ville en faisant appel à l'évêque de Clermont pour obtenir des spécialistes qui fabriquaient des tuyaux en bois (TCCG, VI, 1989a, p. 60).

\section{LES ÉDIFICES DE SPECTACLE}

La fin de l'utilisation des édifices de spectacle a souvent été attribuée à l'émergence du christianisme, pour lequel les cruels jeux de gladiateurs ne seraient plus acceptables, pas plus que les pièces de nature parfois obscène qui se jouaient dans les théâtres. Si les Pères de l'Église ont effectivement condamné les jeux de gladiateurs dans l'amphithéâtre, ces jeux n'ont jamais été interdits par les empereurs chrétiens (Matter, 1990). Augustin, pourtant fervent opposant aux jeux, avoue avoir fréquenté l'amphithéâtre de Thagaste dans sa jeunesse, mais il était encore plus friand du théâtre (Augustin, Enarrationes in psalmos, 147, 7).

$\mathrm{Au}$ Haut-Empire, les jeux du théâtre et de l'amphithéâtre, ainsi que l'entretien de ces édifices, étaient en grande partie pris en charge par les notables locaux, qui, par ces munera, assuraient leur popularité. Le déclin des conseils municipaux dans l'Antiquité tardive ne pouvait que mettre en cause ce système de patronage, en particulier pour les jeux de gladiateurs qui devenaient de moins en moins populaires. Il est probable que, vers la fin $d u \mathrm{IV}^{\mathrm{e}} \mathrm{s}$. ou au début du $\mathrm{V}^{\mathrm{e}} \mathrm{s}$., ces combats de gladiateurs n'aient plus guère été donnés (Ville, 1960; De Voe, 2002). Certes, ils ont parfois été remplacés par des venationes, qui n'employaient que des animaux, mais on peut supposer que le nombre de jeux a diminué progressivement et que plus personne ne voulait ou ne pouvait financer les travaux d'entretien des édifices de spectacle. Seules les courses de chars dans les cirques jouaient encore un rôle dans l'Antiquité tardive, rôle d'ailleurs plus politique que sportif (Cameron, 1976).

Les données littéraires sont peu nombreuses pour le midi de la Gaule. Au milieu du IV ${ }^{\mathrm{e}}$ s., l'épitaphe bien connue de Nymfius, peut-être le propriétaire du domaine de Valentine où cette inscription a été trouvée, montre que ce notable avait donné, durant sa carrière civile, des jeux (munera), sans que l'on sache de quel type de jeux il pouvait s'agir (CIL, XIII, 128; Pailler, 1986). Salvien de Marseille, dans les années 440-450, explique que la récente fin de ces jeux dans la majorité des villes ne témoigne pas pour autant d'une amélioration de la morale chrétienne, mais plutôt d'un manque d'argent pour l'organisation de ces jeux (Du gouvernement de Dieu, VIII, 39-45). On peut cependant se demander si cette explication n'est pas en grande partie due à l'opinion très pessimiste qu'avait le prêtre marseillais de ses compatriotes romains et de son temps. Au début du VI ${ }^{\mathrm{e}}$ s., l'évêque Césaire d'Arles reproche toujours à ses fidèles d'aller plus volontiers aux jeux qu'à l'église et les prévient contre les dangers pour l'âme d'assister aux spectacles cruels (Sermon, 31, 2 ; 61, 3 ; 150, 3). Il incite ses ouailles à ne plus aller au théâtre, dont on sait par ailleurs, qu'il avait commencé à être dépouillé de son décor de marbre durant l'épiscopat de son prédécesseur Hilaire (429-449) (Vie de saint Hilaire, 20).

Le sort des grands édifices de spectacle (théâtre, amphithéâtre, cirque) dépend en grande partie de leur localisation dans la ville de l'Antiquité tardive. Le dépouillement puis la récupération complète de ces édifices est chose courante, puisque ces vastes monuments étaient difficilement réutilisables. Les constructions situées à l'extérieur de l'enceinte de l'Antiquité tardive ont en général entièrement disparu, au point d'avoir été parfois oubliées. C'est le cas notamment pour l'Aquitaine, où les théâtres urbains sont très rares, mais où nous avons quelques indications pour les amphithéâtres. À Agen, où l'abandon de l'amphithéâtre a pu être observé à plusieurs endroits, il semble que la récupération antique a été limitée; en revanche, l'édifice a été remblayé sans doute dès la seconde moitié $d u \mathrm{IV}^{\mathrm{e}} \mathrm{s}$. (Bizot, Fincker, 1992, p. 64). En revanche, à Saintes, on a supposé que l'amphithéâtre a été abandonné puis spolié pour la construction de l'enceinte et la même remarque vaut probablement pour la plupart des autres édifices de cette région (Doreau et al., 1982, p. 19).

En Narbonnaise, la situation est différente, à la fois parce que la plupart des villes étaient déjà fortifiées dès le Haut-Empire et parce que la construction des enceintes « réduites» est plus tardive. Si à Orange, les quelques sondages n'ont pas donné d'indications sur la date et les circonstances de l'abandon de l'amphithéâtre situé à peu de distance de la courtine occidentale de la cité, à Béziers, les travaux récents ont permis de savoir que l'amphithéâtre, situé au sud de la ville dont il est séparé par le tracé supposé de la voie Domitienne, a été abandonné, probablement à la fin du $\mathrm{III}^{\mathrm{e}} \mathrm{s}$., et spolié en grande partie, avant d'être réintégré dans le tissu urbain au XII ${ }^{\mathrm{e}} \mathrm{s}$. (Ginouvez et al., 1995). Pour Narbonne, on a supposé que l'amphithéâtre a été complètement spolié, sans doute dès le IV ${ }^{\mathrm{e}} \mathrm{s}$., " puisque ni Ausone, ni Sidoine n'en parlent» (Dellong, 2002, p. 132). La valeur de cet argument est toutefois limitée, car ce même Ausone ne mentionne pas les Piliers de Tutelle à Bordeaux, dont on sait qu'ils ont été conservés jusqu'au XVII ${ }^{\mathrm{e}} \mathrm{s}$. Dans le cas narbonnais, la situation de l'amphithéâtre par rapport à l'enceinte est plus compliquée, car on ignore la date de 
la construction de l'enceinte tardive, qui exclut cet édifice de spectacles, parce que ce rempart, traditionnellement daté de la fin du III $^{\mathrm{e}}$ s. (Gayraud, 1981, p. 284; Dellong, 2002, p. 147), nous paraît plus récent (Heijmans, 2004a, p. 119-122; voir infra, p. 63-64). On ne peut pas savoir si l'abandon de ce secteur de la ville est le résultat de sa position extra muros ou si, au contraire, il a déterminé le tracé de l'enceinte tardive.

Quelques fouilles récentes des édifices de spectacle nous permettent d'étudier plus en détail les traces de cette occupation tardive, qui ont souvent disparu lors du dégagement de la plupart des monuments encore conservés au XIX ${ }^{\mathrm{e}} \mathrm{s}$.

Ainsi à Arles, le cirque, après avoir peut-être subi des modifications sous Constantin - ce qui témoigne de l'importance de la ville en tant que résidence impériale -, a commencé à être habité au début du $\mathrm{V}^{\mathrm{e}} \mathrm{s}$., ce qui n'a pas empêché qu'il continue à être utilisé pour des jeux (fig. 15). En effet, la dernière attestation des courses de chevaux à Arles date du milieu du VI ${ }^{\mathrm{e}} \mathrm{s}$. Certes, le texte de Procope (Guerre des Goths, VII, 33,5) ne précise pas que ces jeux ont eu lieu dans le cirque, mais c'est à cette même période que l'édifice est complètement spolié après l'abandon de cet habitat (Sintès, 1994, p. 185-189; Heijmans, 2004a, p. 360-365). On le voit encore mieux à Aix-en-Provence, où les fouilles en cours de Núria Nin ont mis au jour dans la partie ouest de la ville, où l'on soupçonnait la présence d'un amphithéâtre (Guyon et al., 1998, p. 257-259), les vestiges d'un vaste théâtre, occupé depuis $\mathrm{le}^{\mathrm{e}} \mathrm{s}$. par un habitat qui se poursuit jusqu'aux $\mathrm{XI}^{\mathrm{e}}-\mathrm{XII}^{\mathrm{e}} \mathrm{s}$. qui est sans doute à mettre en rapport avec la "ville des Tours », qui occupait ce quartier au Moyen Âge (Nin, 2005 et voir infra, p. 43-45).

Comme pour les temples, il peut arriver qu'un édifice extra muros n'ait pas été entièrement spolié, comme à Bordeaux, où l'amphithéâtre dit Palais Gallien semble avoir été incendié vers 276, d'après les anciens sondages de R. Étienne, mais n'a pas été ensuite complètement démonté (Étienne, 1962, p. 193). Même si l'interprétation de ce niveau d'incendie reste discutable, et qu'il ne s'agit peut-être que de l'effondrement et de la décomposition de la charpente, il n'est reste pas moins que l'édifice a relativement bien traversé les siècles.

D’autres édifices, situés plus près du centre des villes, ont été intégrés dans les fortifications et transformés en bastions, comme les amphithéâtres d'Arles, de Nîmes ou de Périgueux. Dans ce dernier cas, le rapport entre l'enceinte tardive et l'amphithéâtre n'est pas encore très clair, mais des éléments de ce dernier ont été utilisés pour la construction du rempart, sans doute au début du IV ${ }^{\mathrm{e}} \mathrm{s}$. (Girardy-Caillat,
1996). Quant à Arles et Nîmes, l'enceinte est plus tardive et ne remonte certainement pas plus haut que le $\mathrm{V}^{\mathrm{e}} \mathrm{s}$; c'est sans doute à partir de ce moment que ces édifices ont abrité, jusqu'à l'époque moderne, un habitat dense, qui leur ont permis d'être assez bien conservés. Quant au théâtre d'Arles, son intégration dans l'enceinte tardive, qui a utilisé son entrée sud comme tour, n'a, en revanche, pas empêché sa disparition presque complète, après une période d'occupation des espaces sous les gradins, sans doute à partir du début $d u V^{\mathrm{e}} \mathrm{s}$. C'est vers la même période qu'à Apt, le théâtre antique, déjà à l'abandon depuis plusieurs siècles, est occupé par des habitats, causant sa destruction complète (Michèle, 2003, p. 227-228), contrairement à Orange, où l'occupation a sans doute également débuté au $\mathrm{V}^{\mathrm{e}} \mathrm{s}$., mais où le théâtre nous est parvenu malgré cela dans un état presque parfait.

$$
\text { * } \quad *
$$

Durant l'Antiquité tardive, les villes du midi de la Gaule ont connu une profonde mutation, qui a varié selon les villes, leur statut et leur prospérité économique, mais qui est également plus ou moins facile à appréhender selon l'avancement de la recherche.

Pour certains aspects, comme le rétrécissement de la surface habitée, cette évolution s'était déjà annoncée au $\mathrm{II}^{\mathrm{e}} \mathrm{s}$. et la « crise » du III ${ }^{\mathrm{e}}$ s. n’a fait qu'accélérer ce mouvement, mais elle ne semble pas avoir affecté les monuments publics durant la majeure partie du $\mathrm{IV}^{\mathrm{e}} \mathrm{s}$., sauf dans le cas des cités du Sud-Ouest qui ont été dotées d'une enceinte dès la fin du III ${ }^{\mathrm{e}} \mathrm{s}$. Ces édifices semblent en effet avoir été entretenus, du moins ceux qui étaient situés au cœur des villes, dont le centre civique n'a pas été touché. Rien ne permet de penser à une dégradation des temples classiques; seule la survie des thermes publics, dont l'entretien était particulièrement coûteux, semble compromise. Il en va autrement, en tout ou en partie, pour les monuments de spectacle situés désormais à l'extérieur de la ville, qu'elle fût ou non remparée, qui ont le plus souvent été abandonnés au cours du IV ${ }^{\mathrm{e}} \mathrm{s}$. Le cirque d'Arles semble être une exception. De même, les centres civiques qui se trouvaient désormais à l'extérieur d'une nouvelle enceinte, comme c'est le cas pour plusieurs villes d'Aquitaine, ont été abandonnés et, avec eux, les autres monuments situés à l'extérieur de l'enceinte, comme les monuments funéraires.

Le véritable changement se produit à la fin $d u \mathrm{IV}^{\mathrm{e}} \mathrm{s}$. et au début $\mathrm{du} \mathrm{V}^{\mathrm{e}} \mathrm{s}$. C'est à ce moment-là que l'on voit les premières installations sur des espaces publics et la transformation de la voirie. Les grands monuments de 


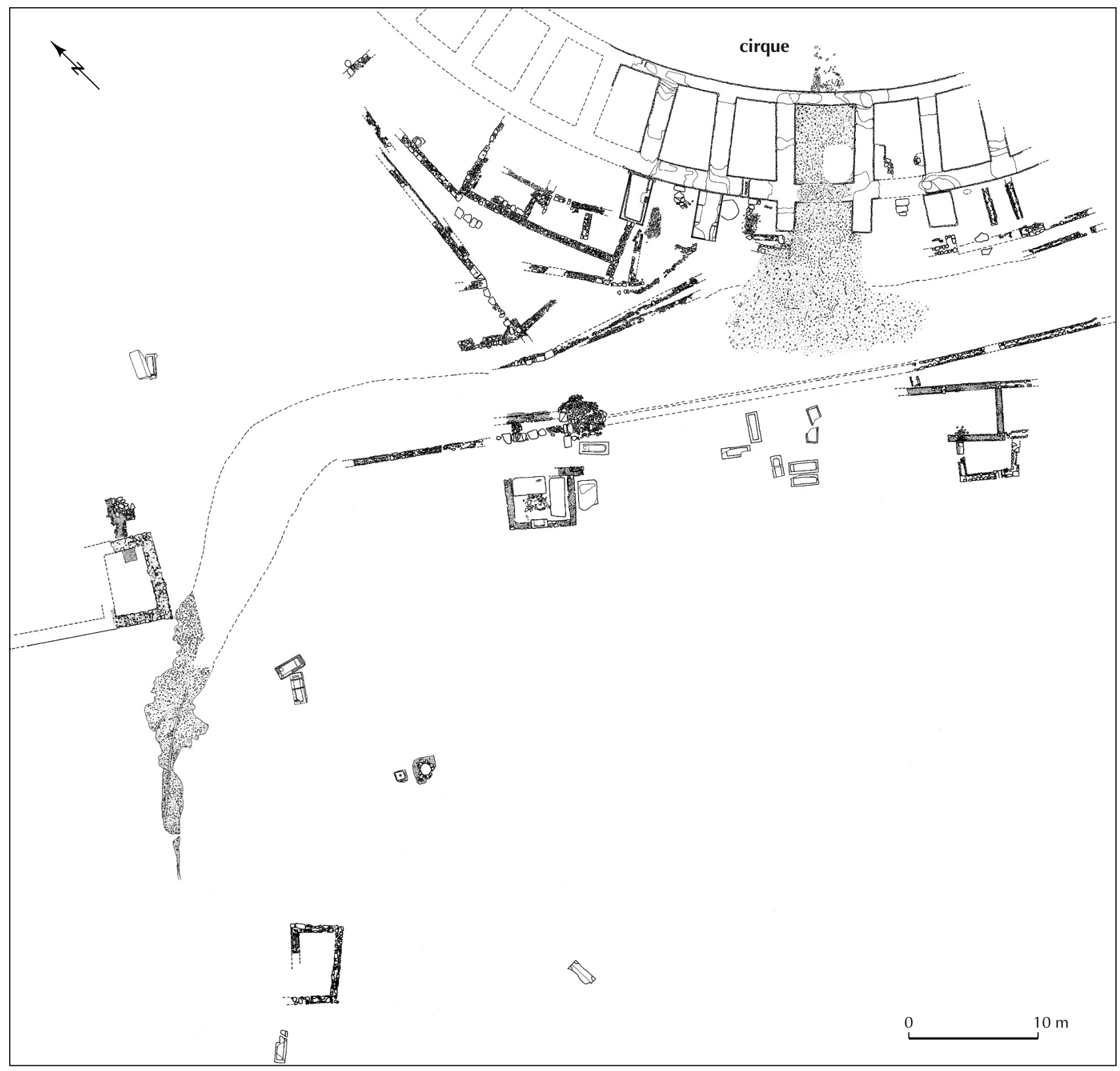

Fig. 15 - Arles (Bouches-du-Rhône). Plan des vestiges tardifs devant l'extrémité ouest du cirque d'Arles (dessin J. Brémond).

spectacles, dont plusieurs étaient déjà abandonnés et qui n’avaient désormais plus d'utilité, sont réutilisés ou démantelés pour la récupération des matériaux de construction. Cette récupération montre cependant qu'il y avait toujours une activité de construction, mais elle était sans doute limitée pour l'essentiel à l'édification des groupes épiscopaux, ainsi qu'à celle des enceintes. Quant aux édifices publics directement liés au centre civique (forum, temple), c'est à partir du $\mathrm{V}^{\mathrm{e}} \mathrm{s}$. que commence leur désaffectation.
À plusieurs reprises, on constate la présence du groupe épiscopal sur l'emplacement du forum ou à proximité, ce qui témoigne de l'influence croissante des Églises dans les villes de l'Antiquité tardive. Étant donné le faible nombre de cas avérés d'un remplacement d'un temple classique par une église, on peut se demander si cette volonté d'occuper le centre civique n'était pas plus politique que religieuse. C'est en effet à partir du $\mathrm{V}^{\mathrm{e}} \mathrm{s}$. que l'évêque, qui était jusqu'alors seulement le chef spirituel de la communauté 


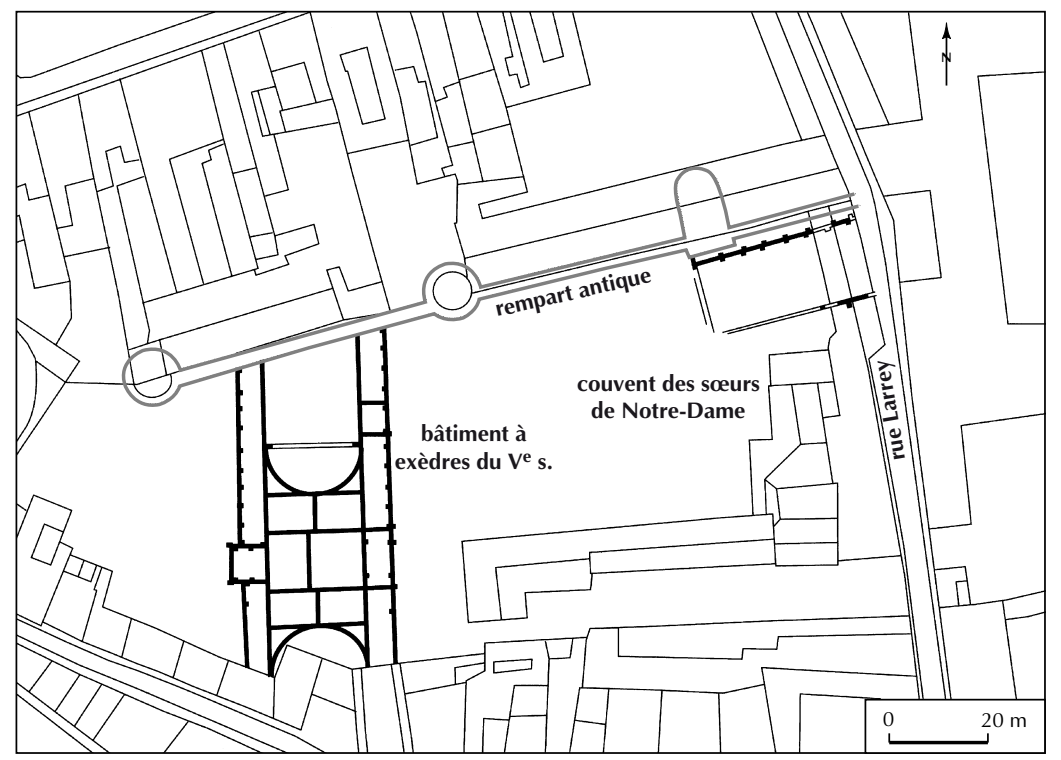

Fig. 16 - Toulouse (Haute-Garonne). Plan du bâtiment tardif mis au jour sur le site de la rue Larrey (d'après Filippo, 1996, p. 26, fig. 3).

chrétienne, devient progressivement, avec la reconnaissance du christianisme comme religion d'État et l'effondrement de l'autorité romaine, le patron de la cité, bien qu'il ne soit qu'une personne privée, sans aucun autre titre. Issu le plus souvent des milieux aristocratiques, surtout en Gaule, il fait naturellement partie des notables locaux et s'occupe de plus en plus également de la gestion séculière, d'autant plus que l'emprise de son évêché est directement calquée sur celle de la cité antique (Beaujard, 1996; Rebillard, Sotinel dir., 1998). On comprend donc aisément que dans ce monde devenu chrétien, les priorités, même pour la gestion de l'espace public, n'étaient plus celles du Haut-Empire, ni celles encore maintenues, avec plus ou moins de succès, au IV $\mathrm{s}$.

Dans ce contexte, il n'est pas surprenant que ce soit dans les grands centres politiques que l'on retrouve encore une activité édilitaire au $\mathrm{V}^{\mathrm{e}} \mathrm{s}$. C'est le cas à Arles, siège du préfet du prétoire des Gaules jusqu'en 476, pour qui on a construit, sans doute aux alentours des années 400, une grande salle de réception, encore remarquablement bien conservée (fig. 13, $\mathrm{n}^{\mathrm{o}}$ 2). Plus tard au cours $d u \mathrm{~V}^{\mathrm{e}}$ s., on agrandit les thermes de Constantin par la construction d'une vaste natatio (fig. 13, no 3) (Heijmans, 1998 et 2004a, p. 160-194 et p. 215-222). C'est également le cas à Toulouse, résidence des rois wisigoths, où une fouille de sauvetage en $1988 \mathrm{a}$ mis au jour un très grand édifice, construit au plus tôt au début du $\mathrm{V}^{\mathrm{e}} \mathrm{s}$. entre le rempart antique et la fortification tardive (fig. 16). L'interprétation de cette grande construction est encore incertaine, bien qu'on ait proposé d'y voir la demeure des rois wisigoths (Filippo, 1996, 2001c; Balmelle, 2001, p. 422). Même si cette interprétation reste difficile à prouver, la construction de ces édifices publics montre que, au-delà de la récupération et de la réappropriation des édifices publics classiques, l'Antiquité tardive a aussi su se montrer inventive quand les conditions politiques, et donc financières, y étaient favorables. 